\title{
Inherited and Environmental Factors Influence Human Monocyte Heterogeneity
}

\author{
Amit A. Patel ${ }^{1 *}$ and Simon Yona ${ }^{2}$ \\ ${ }^{1}$ Division of Medicine, University College London, University of London, London, United Kingdom, ${ }^{2}$ The Institute of Dental \\ Sciences, Hebrew University, Jerusalem, Israel
}

Blood monocytes develop in the bone marrow before being released into the peripheral circulation. The circulating monocyte pool is composed of multiple subsets, each with specialized functions. These cells are recruited to repopulate resident monocytederived cells in the periphery and also to sites of injury. Several extrinsic factors influence the function and quantity of monocytes in the blood. Here, we outline the impact of sex, ethnicity, age, sleep, diet, and exercise on monocyte subsets and their function, highlighting that steady state is not a single physiological condition. A clearer understanding of the relationship between these factors and the immune system may

OPEN ACCESS

Edited by:

Florent Ginhoux

Singapore Immunology Network (A*STAR), Singapore

Reviewed by:

Martin Stacey,

University of Leeds, United Kingdom

Susan Kovats,

Oklahoma Medical Research Foundation, United States

${ }^{*}$ Correspondence: Amit A. Patel amit.patel.12@ucl.ac.uk

Specialty section: This article was submitted to Antigen Presenting Cell Biology,

a section of the journal

Frontiers in Immunology

Received: 09 July 2019

Accepted: 17 October 2019

Published: 07 November 2019

Citation:

Patel AA and Yona S (2019) Inherited and Environmental Factors Influence

Human Monocyte Heterogeneity.

Front. Immunol. 10:2581

doi: 10.3389/fimmu.2019.02581 allow for improved therapeutic strategies.

Keywords: monocyte, macrophage, inflammation, sex, age, diet, exercise, sleep

\section{INTRODUCTION}

Adult blood leukocytes can be separated into two broad categories: lymphoid or myeloid. The lymphoid lineage consists of T, B, innate lymphoid and natural killer (NK) cells, while the myeloid compartment comprises of functionally and morphologically discrete cell types, including mononuclear phagocytes (monocytes and dendritic cells), granulocytes (neutrophils, basophils, and eosinophils), and platelets. Injured or infected tissue releases chemoattractants that rapidly recruit these myeloid cells to the site of injury. Once at the inflamed site, these cells coordinate and carry out key effector functions. Nearly 100 years ago, Alexander Maximow suggested that hematopoiesis was an extremely organized stepwise process arising from a common precursor cell (1). Indeed, hemopoietic stem cells that reside in the bone marrow undergo multiple differentiation stages, becoming progressively more restricted and eventually give rise to a heterogeneous population of leukocytes. Commitment to the myeloid lineage downstream of the common myeloid progenitor (CMP) (2) results in the generation of erythrocytes, platelets, granulocytes, monocytes, and dendritic cells. Nevertheless, several extrinsic factors influence leukocyte generation. Here, we will outline several studies that highlight how age, ethnicity, diet, exercise, sleep, and sex modulate human monocyte numbers under healthy homeostasis.

Circulating blood monocytes were initially believed to be a single population of cells with the potential to repopulate terminally differentiated resident mononuclear phagocytes in the periphery (3). In addition, blood monocytes act as an emergency squad recruited to sites of injury where they perform pro-inflammatory and pro-resolving functions (4-8). Blood monocytes were initially defined by their morphology. Later, with the introduction of flow cytometry, monocytes were shown to express high levels of the lipopolysaccharide (LPS) binding protein receptor, CD14 (9). 
These $\mathrm{CD}^{+} 4^{+}$monocytes were subsequently discovered to be a heterogeneous population that could be further divided into discrete subsets based on CD14 and CD16 (Fc $\gamma$ RIII) expression in humans (10). Monocyte heterogeneity was later observed to be conserved among other species (11). Human CD14 ${ }^{+} \mathrm{CD} 16^{-}$ monocytes, also known as classical monocytes, are analogous to the murine $\mathrm{Ly}_{6} \mathrm{C}^{\mathrm{Hi}} \mathrm{CX}_{3} \mathrm{CR} 1^{\text {int }}$ classical monocytes. Intermediate and non-classical monocytes are identified as $\mathrm{CD} 14^{+}, \mathrm{CD}_{16}{ }^{+}$, and $\mathrm{CD} 14^{\mathrm{lo}} \mathrm{CD} 16^{+}$cells, respectively, where the latter subset mirror Ly6 $\mathrm{C}^{\mathrm{Low}} \mathrm{CX}_{3} \mathrm{CR} 1^{\mathrm{Hi}}$ non-classical monocytes in mice (12, 13). The expression of several membrane adhesion molecules and chemokine receptors can also be used to discriminate between these monocyte subsets (13-15).

Blood monocytes begin their life in the bone marrow, following a similar developmental fate to dendritic cells where they both arise from the macrophage/dendritic cell precursor (MDP) (16). In mice, the MDP was initially proposed to give rise to monocytes and dendritic cells but not granulocytes (17). Downstream of the MDP, the common monocyte progenitor (cMoP) was identified, which gives rise exclusively to classical monocytes (18). The human equivalent of the murine $\mathrm{cMoP}$ was recently identified within the bone marrow granulocytemonocyte progenitors (GMP) fraction (19). This human $\mathrm{cMoP}$ gives rise directly to pre-monocytes, and subsequently, monocytes. Investigations into the developmental stages between the $\mathrm{cMoP}$ and mature monocytes uncovered in mice a Ly6C $^{+}$CXCR4 $^{+}$monocyte subset that resides in the bone marrow termed pre-monocytes (20). These pre-monocytes show proliferative potential and downregulate CXCR4 upon entry into the circulation (20). The egression of murine classical monocytes from the bone marrow follows a circadian rhythm, regulated in part by CXCR4 (20) and the circadian gene Bmall (21). It is widely accepted that classical and non-classical monocyte subsets are related developmentally, with classical monocytes having the potential to give rise to non-classical monocytes over time (14, 22-27) (Figure 1). While recent advances demonstrate monocyte development to be a highly regulated process under steady state, here we summarize the influence of inherited traits and lifestyle choices have on human monocyte homeostasis.

\section{LIFESTYLE AND GENETIC FACTORS AFFECTING MONOCYTES}

Our knowledge concerning the development and function of monocytes and monocyte-derived cells during pathology continues to expand. It is also necessary to appreciate the behavior of these cells under healthy physiological conditions. However, healthy homeostasis is not a solitary state, rather several factors-often overlooked-including sex, diet, exercise, and age influence the immune system. Whether genetic traits prevail over environmental cues regarding the immune response remains a matter of debate $(28,29)$.

The earliest accounts of the cell originate with Robert Hooke's seminal observations in 1665 (30). Cellular analysis began with microscopy, then progressed to flow cytometry and is currently enjoying a renaissance in the form of single-cell RNA analysis. Every advancing stride has led to a greater appreciation regarding the complexity and diversity of monocytes, their subsets, and

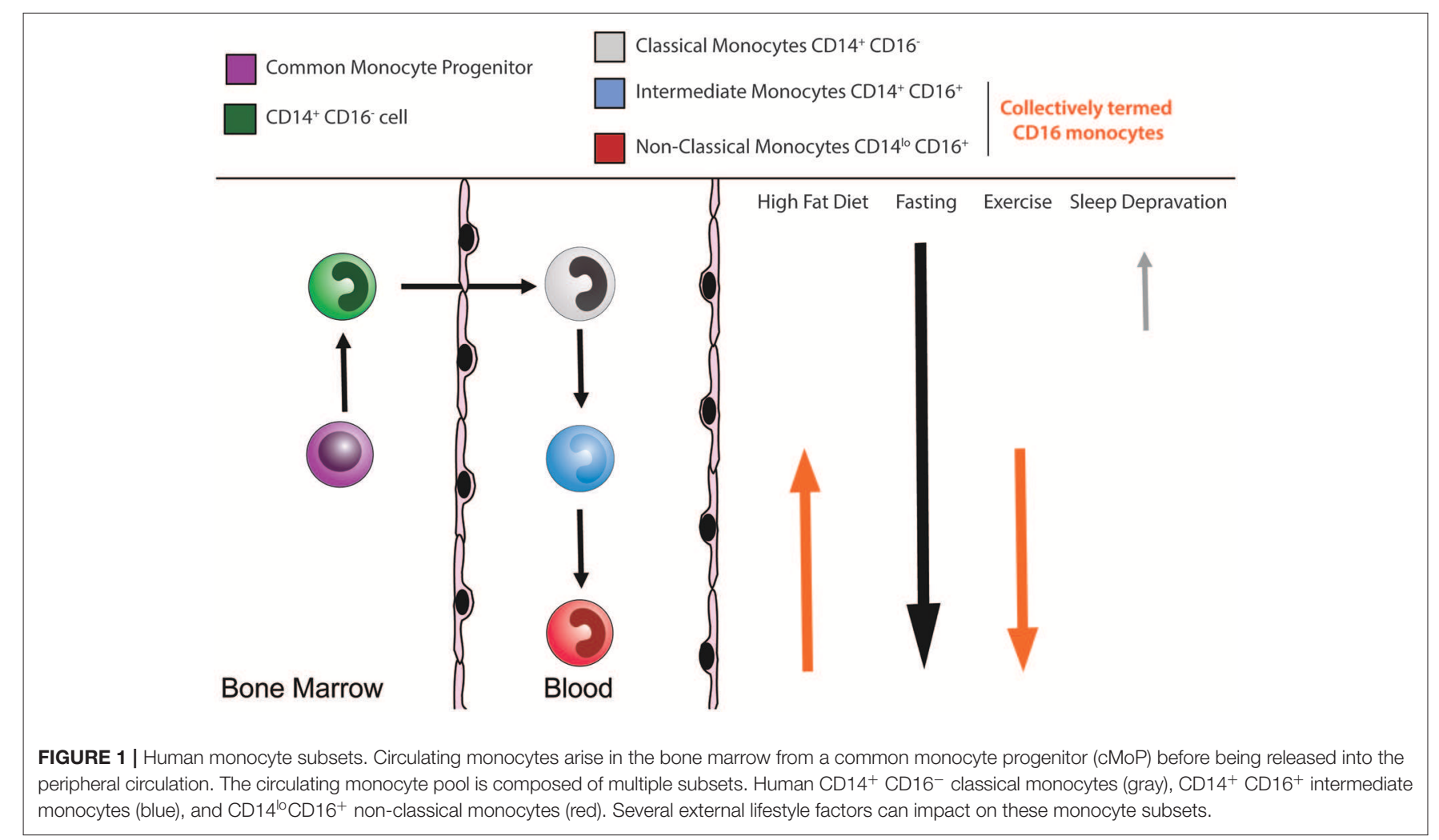


function (31-34). Here, we consider if and how, lifestyle choices imprint on this diversity. This review will focus on our current understanding of human monocyte adaptations observed due to genetic and environmental factors; for a comprehensive review on monocyte biology, see Guilliams et al. (5) and Jakubzick et al. (6).

As a word of caution, the monocyte nomenclature was recently codified by Ziegler-Heitbrock et al. and approved by the International Union of Immunological Societies $(35,36)$. Nevertheless, complexity remains an issue within the historical literature and is further confounded by subsets conveyed sometimes as a proportion of total monocytes or in absolute numbers. Here, we have retold studies as originally described to avoid confusion.

\section{SEX}

Several physiological differences exist between the sexes, the most apparent being their role in reproduction. Another obvious difference is hormone concentration. Over three quarters of patients with autoimmune disease are female (37). Systemic lupus erythematosus (SLE), Sjögren syndrome, fibromyalgia, and rheumatoid arthritis afflict females more than males $(38,39)$, whereas ankylosing spondylitis, vasculitis, and Goodpasture syndrome predominantly occur in males (40). This sexual dimorphism of autoimmunedriven disease begs the question, do male and female immune systems differ?

Under physiological conditions, monocyte counts have consistently been reported to be elevated in males at all stages of life (41-43). This difference is most profound during adolescence (44). Curiously, one study examining over 400 individuals reported that monocytes are higher in Caucasian men than those in women; this difference was absent in the Afro Caribbean population (41). The proportion of non-classical monocytes has also been reported to be different amongst men and women (45). These differences in monocyte subsets may be attributed to the effect of estrogen and other sex hormones. To test this, researchers have turned to the menstrual cycle and menopause. During the menstrual cycle, $17 \beta$-estradiol, and progesterone concentrations are consistently increased during the luteal phase in comparison to the follicular phase. Around 40 years ago, an elevated monocyte count was reported during the luteal phase (46). With our growing knowledge on monocytes, it would be worthwhile to revisit the impact of the menstrual cycle on monocyte subpopulations, especially as the identification of monocytes subsets remains inconsistent throughout the literature. Interestingly, postmenopausal women exhibit lower concentrations of estrogen and tend to have an increased blood monocyte count; nonetheless, following estrogen replacement therapy, monocyte numbers were restored to levels seen in younger females (47). These data suggest an increase in estrogen decreases monocyte numbers, supporting the observation that males tend to have a higher monocyte count. An exception to this would be the increase in monocytes observed during the luteal phase of the menstrual cycle. Together, this may suggest other endogenous factors affect monocyte composition.

Sex differences may become conspicuous in the disease setting. Both healthy males and females have equivalent number of intermediate monocytes; however, only male sarcoidosis patients exhibit an elevated number of intermediate monocytes compared to female sarcoidosis patients who had equivalent numbers to healthy female controls (48).

Sexual dimorphism has been reported in monocyte cytotoxic activity (49) and cytokine production. Male monocytes produce more inflammatory cytokines than females when stimulated with LPS (42); however, female sex hormones were not responsible for this effect as demonstrated in vivo by oral contraceptive use (50). It is important to note that these studies used LPS. PBMCs co-cultured with estrogen had altered expression of TLR3, TLR7, TLR8, TLR9, but not TLR2 and the LPS receptor, TLR4 (51). Therefore, the impact of estrogen on monocyte function may only become apparent in response to particular stimuli. These diverse responses on monocyte function between the sexes are discussed in detail (52). Further studies will help define the cytokines and/or hormones responsible for the divergence observed in monocyte count and function between males and females.

\section{ETHNICITY}

Ethnic diversity is reflected in disease susceptibility across different human populations (53). This has been identified in patients with tuberculosis (TB) infection (54), autoimmune hepatitis (55), and systemic lupus erythematosus (SLE) (56). A groundbreaking study by Nédélec et al. proposes that different environmental pressures on our immune system may explain why African ancestry is associated with a stronger inflammatory response compared to Europeans (57).

Ancestry has been shown to influence leukocyte counts, including neutrophils, lymphocytes, eosinophils, and monocytes $(41,58,59)$. A trans-ethnic meta-analysis study revealed that those of a European ancestry tend to have a higher monocyte count compared to African-American and Japanese individuals (59). As expected, the interplay of ethnicity and other variables is likely to influence monocyte count. Caucasian males were observed to have a significantly higher count compared to African males, while no difference was noted for females (41), demonstrating the interaction of sex and ethnicity.

Regarding individual monocyte subsets, recently, a single study has shown that Caucasian populations tend to have a higher percentage of classical monocytes and conversely a lower percentage of non-classical monocytes than those sampled from an African population (58). In this study, Caucasians had a trend for increased $\mathrm{CX}_{3} \mathrm{CR} 1$ expression; this could explain the difference in monocyte proportions. $\mathrm{CX}_{3} \mathrm{CR} 1$ is involved in non-classical monocyte retention to the endothelium (60, 61 ), as well as a survival factor for this monocyte population (62); therefore, this increased expression might result in lower representation of free circulating non-classical monocytes due to their increased adherence. Of note, ethnic differences 
are possibly reflected in monocyte function, as monocytederived cells from Filipino, Chinese, and non-Hispanic whites challenged with Mycobacterium tuberculosis produced varying quantities of cytokines from 137 volunteers ( $>44$ from each background) (63). As ethnicity can influence the immune response, this may implicate the need for a more personalized take regarding therapeutics.

\section{DIET}

Diet varies from individual to individual-from what they eat to the quantity and frequency. A balanced diet is a basic requirement for a healthy lifestyle. This fine balance goes awry during surplus calorie intake, which contributes to many diseases, including atherosclerosis, type 2 diabetes, and nonalcoholic fatty liver disease (NAFLD). These western diet-related diseases are associated with systemic chronic inflammation (64).

Monocytes play a central role in several diet-related pathologies. In recent years, it has become evident that a highfat western diet triggers a number of functional modifications in monocytes. Mice fed a western diet for 4 weeks led to an elevation in circulating and splenic monocytes compared to those fed on a standard chow diet (65). Further examination into how a western diet prompts myelopoiesis was described to be due to an increase in GMP in the bone marrow that also functionally reprograms myeloid cells through NLRP3. Upon reverting to a chow diet, monocyte numbers returned to baseline, although an increased activation status became imprinted in classical monocytes. Collectively, these data suggest that diet leads to innate immune training within the monocyte pool in mice. In a human study, $3 \mathrm{~h}$ after the consumption of a high-fat (McDonald's) meal resulted in an increased monocyte count, specifically an elevation in non-classical monocytes (66). Similarly, this observation is consistent with a study where $\mathrm{CD} 6^{+}$monocytes (intermediate and non-classical) positively correlated with increased body weight (67). Interestingly, in these subjects, dietary intervention or gastric bypass surgery resulted in a decrease in the absolute number and percentage of these cells (67). Immediately following a high-fat meal, Khan et al. demonstrated a higher percentage of lipid-laden monocytes (66). These foamy monocytes increase their expression of CD11c, which is thought to lead to monocyte arrest via vascular cell adhesion protein (VCAM-1). While these are short-term effects, recurrent chronic exposure of a high-fat diet may lead to dietrelated diseases.

Although a high-fat dietary intake increases peripheral monocyte numbers, the opposite is true in fasted individuals (68). Dietary restriction for $19 \mathrm{~h}$ in humans or $4 \mathrm{~h}$ in mice led to a significant reduction in both circulating classical and non-classical monocytes. This reduction in blood monocytes was due to the inhibition of monocyte egress from the bone marrow. This elegant study from the Merad group demonstrated that carbohydrate and protein consumption rescues monocyte numbers via the liver activated protein kinase-peroxisome proliferator-activated receptor alpha (AMPKPPAR $\alpha$ ) pathway that regulates the monocyte chemoattractant protein, CCL2 (68). Furthermore, dietary restriction during experimental autoimmune encephalomyelitis (EAE), a mouse model of multiple sclerosis, improved disease outcome and reduced myeloid cell infiltrate compared to animals with access to food ad libitum $(68,69)$. Similar findings have been observed in humans, where fasting diets have shown to improve the quality of life for patients with multiple sclerosis $(69,70)$.

In an experimental setting, a single macronutrient or micronutrient alters the monocyte composition and function. It is more realistic that it is a combination of several dietary nutrients in varying amounts that will alter the phenotype of monocytes.

\section{SLEEP/WAKE CYCLE}

Cortisol is the archetypical neuroendocrine anti-inflammatory hormone that regulates immune cell gene expression $(71,72)$. Cortisol follows a diurnal pattern where it peaks $30 \mathrm{~min}$ after waking in the morning and falls throughout the day. Cortisol is the endogenous member of the glucocorticoid family of immunosuppressive and anti-inflammatory drugs that acts on many leukocytes, including monocytes, where they induce a rapid decrease in circulating monocytes (73). Therefore, it would be interesting to know if monocytes also follow a diurnal pattern.

Meuret et al. performed to the best of our knowledge one of the earliest studies on monocyte kinetics. Meuret and colleagues observed a monocyte cycle occurring around every 5 days in humans. They proposed this due to a homeostatic loop within the bone marrow with transit time being the prevailing factor (74). Recently, we observed a population of $\mathrm{CD} 14^{+} \mathrm{CD} 16^{-}$cells resembling classical monocytes that reside in the human bone marrow (14); these cells exhibit a postmitotic dwell period of $\sim 1.5-2$ days before being released into the circulation $(14,75)$. It is possible that this maturation period, in addition to the time taken to differentiate into these cells, and the regulatory signals account for this monocyte cycle.

While long-term monocyte oscillations have been described, diurnal patterns are also present in both mice $(20,21)$ and humans, where monocyte numbers decrease during sleep and begin to gradually rise upon waking $(76,77)$. Cuesta et al. were able to stratify individuals into two categories, one where monocyte numbers peak during the morning, and another group where the monocyte count peaked in the evening (77). The reason behind the existence of these two groups remains unclear-all subjects except one were male, of a similar age who maintained comparable levels of activity, equivalent calorie intake, and exposure to light. In mice, CXCR4 and Bmal1 regulate the circadian rhythm of circulating monocytes $(20,21)$.

Functional changes such as cytokine production, surface membrane protein expression, and phagocytic ability have been reported to follow a diurnal pattern (77-80). Cuesta et al. conclude that cytokine production follows a bimodal rhythm, where monocytes are more responsive at night, whereas during the day, a higher production results from the increased numbers of monocytes (77). In addition, while TLR1, 2, 4, and 9 expression does not differ throughout the day, activation of these receptors 
results in the dampened expression of costimulatory molecules in the morning (78).

Sleep-deprived individuals who remain awake during the night gain a higher monocyte count compared to those who slept at this period (76). Congruent with these findings, mice with disrupted sleep also have an increase in peripheral blood classical monocytes (81). Uninterrupted sleep enables the release of hypocretin from the hypothalamus, which in turn regulates CSF1 production from bone marrow pre-neutrophils regulating monopoiesis (81). Although an increase in monocyte count occurs during sleep deprivation, a diurnal oscillation pattern remains (82). Furthermore, in night shift workers who are active at night, no phase shift was detected in monocyte numbers (77). Taken together, these studies establish that both internal and external body clocks influence monocyte behavior and emphasize the importance of appropriate time controls when conducting experiments.

Circannual or seasonal rhythms have also been detected in monocyte function. Monocyte counts remain stable throughout the year, yet phagocytosis, cytokine production, and prostaglandin metabolism vary $(83,84)$. Specifically, a higher proportion of monocytes phagocytose in winter compared to spring and autumn. In response to LPS, monocytes isolated during the autumnal period produce lower concentrations of both inflammatory [tumor necrosis factor (TNF)- $\alpha$ and interleukin (IL)-6] and anti-inflammatory (IL-10) cytokines. There are several possible explanations for the fluctuation in human monocyte function throughout the year; seasonal changes may be influenced by a myriad of factors including increased periods in crowded places (i.e., indoor contagion theory), reduced exposure to sunlight (vitamin D deficiency), or even temperature changes. Despite the reason for these changes, these seasonal changes may be relevant when performing long-term clinical trials and should be taken into consideration.

\section{EXERCISE}

Over 120 years ago, Schulte described that exercise impacts the human immune system and induces leukocytosis (85). Therefore, the question arises, do monocytes fluctuate during exercise? Studies have reported a rise in intermediate and non-classical monocytes immediately following exercise (86), while others have described a change in classical monocytes (80), classical and non-classical monocytes (87), and even all three populations $(88,89)$. At first glance, this can appear confusing however; not all exercise is equal as the intensity, duration, and the type of exercise (aerobic or anaerobic) influence monocytes (90) and may account for these different findings. Maximal oxygen consumption, a readout of an individual's fitness, negatively correlated with the percentage change in intermediate monocytes (89). Therefore, the larger the maximal oxygen consumption, the smaller the percentage change in intermediate monocytes. The majority of non-classical monocytes are constantly crawling on the endothelium in an LFA1-dependent manner $(60,61)$. This may be overcome during intense exercise by the increase in shear blood flow. Patrolling monocytes that previously were crawling on endothelial cells are now able to be isolated, providing a more genuine picture of blood monocyte subsets. While these effects are transient, long-term alterations in monocyte composition have also been observed following exercise. A 12-weeks exercise regime reduced the percentage of $\mathrm{CD}^{+} 6^{+}$monocytes (91). This decline in intermediate monocytes could be associated with fat loss (67), as described above. In addition, TNF- $\alpha$ production was significantly reduced following this 12 -weeks exercise program, while monocyte phagocytosis increased, suggesting that longterm exercise may promote a more anti-inflammatory monocyte while improving its phagocytic capacity.

\section{AGE}

At birth, monocytes are three times higher compared with those of adults (92). Christensen and colleagues analyzed over 63,000 hospital records and found that monocyte counts increase linearly with gestational age (93). This monocyte expansion continued into the first 2 weeks after birth. As an organism ages, so does its immune system. The term inflamm-aging was coined by Franceschi 20 years ago (94) to describe the progressive increase in the organism's proinflammatory status as it matures. Inflamm-aging includes adaptive immunity, immunosenescence, and dysregulation of the innate immune response. While some studies have reported no changes in the mononuclear phagocyte count in different age cohorts $(43,95)$, others have noticed a decrease in dendritic cells (96) and an increase in monocyte subsets in the older aged cohort $(45,96-$ 99), particularly in intermediate and non-classical monocytes. Plasma CCL2 is elevated in older individuals (99), which may result in monocyte mobilization from the bone marrow, resetting the dynamic equilibrium of blood monocyte subsets. However, in advanced old age (81-100 years), fewer classical and intermediate monocytes have been detected (100).

Coincidently, $\mathrm{CD}^{+} 6^{+}$monocytes have been reported to expand in inflammatory conditions, also increased in older individuals. These monocytes, in particular, non-classical monocytes, produce higher basal levels of TNF- $\alpha$ and is thought to account for the increase in plasma TNF- $\alpha$ levels in aged individuals $(45,97,101)$. A consequence of this inflammatory environment results in dysregulated innate immunity, such as impaired phagocytosis (45). The pharmacological blockade of TNF- $\alpha$ in aged mice improved bacterial clearance and returned classical monocytes to levels in young mice (102). Therefore, the accumulation of monocytes in the elderly may account for age-related inflammatory conditions. While plasma TNF- $\alpha$ changes with age, hormonal changes also occur. Therefore, it is important to consider age and environmental and genetic factors, as all interact with the immune system. There are as many "young" 85-year-olds running marathons as "old" sedentary 75-year-olds.

\section{CONCLUSION}

Recently, new monocyte subsets have been described in mice $(35,103-105)$ and humans $(32,34)$. The identification of 
these new populations demonstrates how recent technological advances have changed the mononuclear phagocyte landscape. Future insight to these subsets will provide therapeutic strategies to enhance these cells when they are beneficial and block the detrimental effects. In the quest for novel therapeutics, it is critical to remember how sex and environmental and lifestyle choices lead to physiological variations within a leukocyte population as discussed here for monocytes. It is important to examine these variables from a holistic stance using defined objective criteria to avoid bias that may have crept into previous studies. Historically, research into the impact of lifestyle choices was performed on a limited cohort with certain subjective evaluations. The adoption of electronic health records will provide greater insight into how sex and environmental and lifestyle choices

\section{REFERENCES}

1. Maximow AA. Relation of blood cells to connective tissues and endothelium. Physiol Rev. (1924) 4:533-63. doi: 10.1152/physrev.1924.4.4.533

2. Akashi K, Traver D, Miyamoto T, Weissman IL. A clonogenic common myeloid progenitor that gives rise to all myeloid lineages. Nature. (2000) 404:193-7. doi: 10.1038/350 04599

3. van Furth R, Cohn ZA, Hirsch JG, Humphrey JH, Spector WG, Langevoort HL. The mononuclear phagocyte system: a new classification of macrophages, monocytes, and their precursor cells. Bull World Health Organ. (1972) 46:845-52.

4. Ebert RH, Florey HW. The extravascular development of the monocyte observed in vivo. Br J Exp Pathol. (1939) 20:342.

5. Guilliams M, Mildner A, Yona S. Developmental and functional heterogeneity of monocytes. Immunity. (2018) 49:595-613. doi: 10.1016/j.immuni.2018.10.005

6. Jakubzick CV, Randolph GJ, Henson PM. Monocyte differentiation and antigen-presenting functions. Nat Rev Immunol. (2017) 17:349-62. doi: $10.1038 /$ nri.2017.28

7. Patel AA, Yona S. Phagocyte development. In: eLS. Chichester, UK: John Wiley \& Sons, Ltd. (2018). p. 1-13. doi: 10.1002/9780470015902.a0001218.pub2

8. Yona S, Gordon S. From the reticuloendothelial to mononuclear phagocyte system - the unaccounted years. Front Immunol. (2015) 6:328. doi: 10.3389/fimmu.2015.00328

9. Griffin JD, Ritz J, Nadler LM, Schlossman SF. Expression of myeloid differentiation antigens on normal and malignant myeloid cells. J Clin Invest. (1981) 68:932-41. doi: 10.1172/JCI110348

10. Passlick B, Flieger D, Ziegler-Heitbrock HW. Identification and characterization of a novel monocyte subpopulation in human peripheral blood. Blood. (1989) 74:2527-34. doi: 10.1182/blood.V74.7.2527.2527

11. Ziegler-Heitbrock L. Monocyte subsets in man and other species. Cell Immunol. (2014) 289:135-9. doi: 10.1016/j.cellimm.2014. 03.019

12. Geissmann F, Jung S, Littman DR. Blood monocytes consist of two principal subsets with distinct migratory properties. Immunity. (2003) 19:71-82. doi: 10.1016/S1074-7613(03)00174-2

13. Ingersoll MA, Spanbroek R, Lottaz C, Gautier EL, Frankenberger M, Hoffmann R, et al. Comparison of gene expression profiles between human and mouse monocyte subsets. Blood. (2010) 115:e10-9. doi: 10.1182/blood-2009-07-235028

14. Patel AA, Zhang Y, Fullerton JN, Boelen L, Rongvaux A, Maini AA, et al. The fate and lifespan of human monocyte subsets in steady state and systemic inflammation. J Exp Med. (2017) 214:1913-23. doi: 10.1084/jem.20 170355 impact monocytes and additional leukocyte populations on a previously unimaginable scale.

\section{AUTHOR CONTRIBUTIONS}

All authors listed have made a substantial, direct and intellectual contribution to the work, and approved it for publication.

\section{FUNDING}

AP was supported by an EPSRC studentship.

\section{ACKNOWLEDGMENTS}

We apologize to our colleagues whose work we were unable to cite due to space restrictions.

15. Wong KL, Tai JJ-Y, Wong W-C, Han H, Sem X, Yeap W-H, et al. Gene expression profiling reveals the defining features of the classical, intermediate, and non-classical human monocyte subsets. Blood. (2011) 118:e16-31. doi: 10.1182/blood-2010-12-326355

16. Lee J, Breton G, Oliveira TYK, Zhou YJ, Aljoufi A, Puhr S, et al. Restricted dendritic cell and monocyte progenitors in human cord blood and bone marrow. J Exp Med. (2015) 212:385-99. doi: 10.1084/jem.20141442

17. Fogg DK, Sibon C, Miled C, Jung S, Aucouturier P, Littman DR, et al. A clonogenic bone marrow progenitor specific for macrophages and dendritic cells. Science. (2006) 311:83-7. doi: 10.1126/science.1117729

18. Hettinger J, Richards DM, Hansson J, Barra MM, Joschko A-CC, Krijgsveld J, et al. Origin of monocytes and macrophages in a committed progenitor. Nat Immunol. (2013) 14:821-30. doi: 10.1038/ni.2638

19. Kawamura S, Onai N, Miya F, Sato T, Tsunoda T, Kurabayashi K, et al. Identification of a human clonogenic progenitor with strict monocyte differentiation potential: a counterpart of mouse cMoPs. Immunity. (2017) 46:835-48.e4. doi: 10.1016/j.immuni.2017.04.019

20. Chong SZ, Evrard M, Devi S, Chen J, Lim JY, See P, et al. CXCR4 identifies transitional bone marrow premonocytes that replenish the mature monocyte pool for peripheral responses. J Exp Med. (2016) 213:2293. doi: 10.1084/jem.20160800

21. Nguyen KD, Fentress SJ, Qiu Y, Yun K, Cox JS, Chawla A. Circadian gene Bmall regulates diurnal oscillations of Ly6Chi inflammatory monocytes. Science. (2013) 341:1483-8. doi: 10.1126/science.1240636

22. Bianchini M, Duchêne J, Santovito D, Schloss MJ, Evrard M, Winkels $\mathrm{H}$, et al. PD-L1 expression on nonclassical monocytes reveals their origin and immunoregulatory function. Sci Immunol. (2019) 4:eaar3054. doi: 10.1126/sciimmunol.aar3054

23. Gamrekelashvili J, Giagnorio R, Jussofie J, Soehnlein O, Duchene J, Briseño CG, et al. Regulation of monocyte cell fate by blood vessels mediated by Notch signalling. Nat Commun. (2016) 7:12597. doi: 10.1038/ncomms12597

24. Mildner A, Schönheit J, Giladi A, David E, Lara-Astiaso D, Lorenzo-Vivas $\mathrm{E}$, et al. Genomic characterization of murine monocytes reveals C/EBP $\beta$ transcription factor dependence of Ly6C - cells. Immunity. (2017) 46:84962.e7. doi: 10.1016/j.immuni.2017.04.018

25. Sunderkötter C, Nikolic T, Dillon MJ, van Rooijen N, Stehling M, Drevets DA, et al. Subpopulations of mouse blood monocytes differ in maturation stage and inflammatory response. J Immunol. (2004) 172:4410-7. doi: 10.4049/jimmunol.172.7.4410

26. Varol C, Landsman L, Fogg DK, Greenshtein L, Gildor B, Margalit R, et al. Monocytes give rise to mucosal, but not splenic, conventional dendritic cells. J Exp Med. (2007) 204:171-80. doi: 10.1084/jem.200 61011

27. Yona S, Kim KW, Wolf Y, Mildner A, Varol D, Breker M, et al. Fate mapping reveals origins and dynamics of monocytes and tissue macrophages under homeostasis. Immunity. (2013) 38:79-91. doi: 10.1016/j.immuni.2012.12.001 
28. Brodin P, Jojic V, Gao T, Bhattacharya S, Angel CJL, Furman D, et al. Variation in the human immune system is largely driven by non-heritable influences. Cell. (2015) 160:37-47. doi: 10.1016/j.cell.2014.12.020

29. Mangino M, Roederer M, Beddall MH, Nestle FO, Spector TD. Innate and adaptive immune traits are differentially affected by genetic and environmental factors. Nat Commun. (2017) 8:13850. doi: $10.1038 /$ ncomms 13850

30. Hooke R. Micrographia: or Some Physiological Descriptions of Minute Bodies Made by Magnifying Glasses. With Observations and Inquiries Thereupon. London: Jo. Martyn and Ja. Alleftry. (1665). doi: 10.5962/bhl.title.904

31. Cytlak U, Resteu A, Pagan S, Green K, Milne P, Maisuria S, et al. Differential IRF8 requirement defines two pathways of dendritic cell development in humans. Immunity. (2019) 56. doi: 10.2139/ssrn.3365028

32. Günther $\mathrm{P}$, Cirovic $\mathrm{B}$, Baßler $\mathrm{K}$, Händler $\mathrm{K}$, Becker $\mathrm{M}$, Dutertre $\mathrm{CA}$, et al. A rule-based data-informed cellular consensus map of the human mononuclear phagocyte cell space. bioRxiv. (2019). bioRxiv:658179. doi: $10.1101 / 658179$

33. See P, Dutertre C-A, Chen J, Günther P, McGovern N, Irac SE, et al. Mapping the human DC lineage through the integration of high-dimensional techniques. Science. (2017) 356:eaag3009. doi: 10.1126/science.aag3009

34. Villani A-C, Satija R, Reynolds G, Sarkizova S, Shekhar K, Fletcher J, et al. Single-cell RNA-seq reveals new types of human blood dendritic cells, monocytes, and progenitors. Science. (2017) 356:eaah4573. doi: $10.1126 /$ science.aah4573

35. Guilliams M, Ginhoux F, Jakubzick C, Naik SH, Onai N, Schraml BU, et al. Dendritic cells, monocytes and macrophages: a unified nomenclature based on ontogeny. Nat Rev Immunol. (2014) 14:1-8. doi: 10.1038/nri3712

36. Ziegler-Heitbrock L, Ancuta P, Crowe S, Dalod M, Grau V, Hart $\mathrm{DN}$, et al. Nomenclature of monocytes and dendritic cells in blood. Blood. (2010) 116:e74-80. doi: 10.1182/blood-2010-02-2 58558

37. Fairweather D, Frisancho-Kiss S, Rose NR. Sex differences in autoimmune disease from a pathological perspective. Am J Pathol. (2008) 173:600-9. doi: 10.2353/ajpath.2008.071008

38. Regitz-Zagrosek V. Sex and gender differences in health. Sci Soc Series Sex Sci EMBO Rep. (2012) 13:596-603. doi: 10.1038/embor.2012.87

39. Whitacre CC, Reingold SC, O'Looney PA. A gender gap in autoimmunity. Science. (1999) 283:1277-8. doi: 10.1126/science.283.5406.1277

40. Fish EN. The X-files in immunity: sex-based differences predispose immune responses. Nat Rev Immunol. (2008) 8:737-44. doi: 10.1038/nri2394

41. Bain BJ. Ethnic and sex differences in the total and differential white cell count and platelet count Samples were taken into an evacuated tube containing dry K2 EDTA and a full blood count, including an automated differential count, was performed on a Bayer-Techn. J Clin Pathol. (1996) 49:664-6. doi: 10.1136/jcp.4 9.8.664

42. Bouman A, Schipper M, Heineman MJ, Faas MM. Gender difference in the non-specific and specific immune response in humans. Am J Reprod Immunol. (2004) 52:19-26. doi: 10.1111/j.1600-0897.2004.0 0177.x

43. Chen Y, Zhang Y, Zhao G, Chen C, Yang P, Ye S, et al. Difference in leukocyte composition between women before and after menopausal age, and distinct sexual dimorphism. PLoS ONE. (2016) 11:e0162953. doi: 10.1371/journal.pone.01 62953

44. Valiathan R, Ashman M, Asthana D. Effects of ageing on the immune system: infants to elderly. Scand J Immunol. (2016) 83:255-66. doi: 10.1111/sji. 12413

45. Hearps AC, Martin GE, Angelovich TA, Cheng WJ, Maisa A, Landay AL, et al. Aging is associated with chronic innate immune activation and dysregulation of monocyte phenotype and function. Aging Cell. (2012) 11:867-75. doi: 10.1111/j.1474-9726.2012.0 0851.x

46. Mathur S, Mathur RS, Goust JM, Williamson HO, Fudenberg HH. Cyclic variations in white cell subpopulations in the human menstrual cycle: correlations with progesterone and estradiol. Clin Immunol Immunopathol. (1979) 13:246-53. doi: 10.1016/0090-1229(79)9 0069-2
47. Ben-Hur H, Mor G, Insler V, Blickstein I, Amir-Zaltsman Y, Sharp $A$, et al. Menopause is associated with a significant increase in blood monocyte number and a relative decrease in the expression of estrogen receptors in human peripheral monocytes. Am J Reprod Immunol. (1995) 34:363-9. doi: 10.1111/j.1600-0897.1995.tb0 0965.x

48. Hofer TP, Zawada AM, Frankenberger M, Skokann K, Satzl AA, Gesierich $\mathrm{W}$, et al. slan-defined subsets of CD16-positive monocytes: impact of granulomatous inflammation and M-CSF receptor mutation. Blood. (2015) 126:2601-10. doi: 10.1182/blood-2015-06-651331

49. Koopman MG, Fleer A, vd Schaaf DB, van der Meulen FW, von dem Borne AE, and Engelfriet CP. Male-female differences in the cytotoxic activity of human monocytes in vitro. Clin Lab Haematol. (1981) 3:45-50. doi: 10.1111/j.1365-2257.1981.tb01308.x

50. Bouman A, Schipper M, Heineman MJ, Faas M. 17 $\beta$-Estradiol and progesterone do not influence the production of cytokines from lipopolysaccharide-stimulated monocytes in humans. Fertil Steril. (2004) 82:1212-9. doi: 10.1016/j.fertnstert.2004. 05.072

51. Young NA, Wu L-C, Burd CJ, Friedman AK, Kaffenberger BH, Rajaram MVS, et al. Estrogen modulation of endosome-associated toll-like receptor 8: an IFN $\alpha$-independent mechanism of sex-bias in systemic lupus erythematosus. Clin Immunol. (2014) 151:66-77. doi: 10.1016/j.clim.2014.01.006

52. Bouman A, Jan Heineman M, Faas MM. Sex hormones and the immune response in humans. Hum Reprod Update. (2005) 11:411-23. doi: 10.1093/humupd/dmi008

53. Pennington R, Gatenbee C, Kennedy B, Harpending H, Cochran G. Group differences in proneness to inflammation. Infect Genet Evol. (2009) 9:1371-80. doi: 10.1016/j.meegid.2009. 09.017

54. Coussens AK, Wilkinson RJ, Nikolayevskyy V, Elkington PT, Hanifa $\mathrm{Y}$, Islam $\mathrm{K}$, et al. Ethnic variation in inflammatory profile in tuberculosis. PLoS Pathog. (2013) 9:e1003468. doi: 10.1371/journal.ppat.10 03468

55. Wong RJ, Gish R, Frederick T, Bzowej N, Frenette C. The impact of race/ethnicity on the clinical epidemiology of autoimmune hepatitis. J Clin Gastroenterol. (2012) 46:155-61. doi: 10.1097/MCG.0b013e318228b781

56. Gómez-Puerta JA, Barbhaiya $\mathrm{M}$, Guan $\mathrm{H}$, Feldman $\mathrm{CH}$, Alarcón GS, Costenbader KH. Racial/ethnic variation in allcause mortality among united states medicaid recipients with systemic lupus erythematosus: a hispanic and asian paradox. Arthritis Rheumatol. (2015) 67:752-60. doi: 10.1002/art. 38981

57. Nédélec Y, Sanz J, Baharian G, Szpiech ZA, Pacis A, Dumaine A, et al. Genetic ancestry and natural selection drive population differences in immune responses to pathogens. Cell. (2016) 167:657-69.e21. doi: 10.1016/j.cell.2016. 09.025

58. Appleby LJ, Nausch N, Midzi N, Mduluza T, Allen JE, Mutapi F. Sources of heterogeneity in human monocyte subsets. Immunol Lett. (2013) 152:32-41. doi: 10.1016/j.imlet.2013. 03.004

59. Keller MF, Reiner AP, Okada Y, van Rooij FJA, Johnson AD, Chen M-H, et al. Trans-ethnic meta-analysis of white blood cell phenotypes. Hum Mol Genet. (2014) 23:6944-60. doi: 10.1093/hmg/ddu401

60. Auffray C, Fogg D, Garfa M, Elain G, Join-Lambert O, Kayal S, et al. Monitoring of blood vessels and tissues by a population of monocytes with patrolling behavior. Science. (2007) 317:666-70. doi: 10.1126/science.11 42883

61. Carlin LM, Stamatiades EG, Auffray C, Hanna RN, Glover L, Vizcay-Barrena G, et al. Nr4a1-dependent Ly6Clow monocytes monitor endothelial cells and orchestrate their disposal. Cell. (2013) 153:362-75. doi: 10.1016/j.cell.2013. 03.010

62. Landsman L, Bar-On L, Zernecke A, Kim K-W, Krauthgamer $\mathrm{R}$, Shagdarsuren $\mathrm{E}$, et al. CX3CR1 is required for monocyte homeostasis and atherogenesis by promoting cell survival. Blood. (2009) 113:963-72. doi: 10.1182/blood-2008-07-1 70787 
63. Nahid P, Jarlsberg LG, Kato-Maeda M, Segal MR, Osmond $\mathrm{DH}$, Gagneux $\mathrm{S}$, et al. Interplay of strain and race/ethnicity in the innate immune response to M. tuberculosis. PLoS ONE. (2018) 13:e0195392. doi: 10.1371/journal.pone.01 95392

64. Lumeng CN, Saltiel AR. Inflammatory links between obesity and metabolic disease. J Clin Invest. (2011) 121:2111-7. doi: 10.1172/JCI 57132

65. Christ A, Gü P, Lauterbach MAR, Netea MG, Schultze JL, Correspondence L. Western diet triggers NLRP3-dependent innate immune reprogramming. Cell. (2018) 172:162-8.e14. doi: 10.1016/j.cell.2017. 12.013

66. Khan IM, Pokharel Y, Dadu RT, Lewis DE, Hoogeveen RC, Wu H, et al. Postprandial monocyte activation in individuals with metabolic syndrome. J Clin Endocrinol Metab. (2016) 101:4195-204. doi: 10.1210/jc.2 016-2732

67. Poitou C, Dalmas E, Renovato M, Benhamo V, Hajduch F, Abdennour $\mathrm{M}$, et al. $\mathrm{CD} 14^{\mathrm{dim}} \mathrm{CD}^{\mathrm{di}} 6^{+}$and $\mathrm{CD}_{14}^{+} \mathrm{CD}^{+} 6^{+}$ monocytes in obesity and during weight loss. Arterioscler Thromb Vasc Biol. (2011) 31:2322-30. doi: 10.1161/ATVBAHA.111.2 30979

68. Jordan S, Tung N, Casanova-Acebes M, Chang C, Cantoni C, Zhang D, et al. Dietary intake regulates the circulating inflammatory monocyte pool. BioRxiv. (2019) 178:1102-14.e17. doi: 10.1016/j.cell.2019. 07.050

69. Cignarella F, Cantoni C, Ghezzi L, Salter A, Dorsett Y, Chen L, et al. Intermittent fasting confers protection in CNS autoimmunity by altering the gut microbiota. Cell Metab. (2018) 27:1222-35.e6. doi: 10.1016/j.cmet.2018.05.006

70. Choi IY, Piccio L, Childress P, Bollman B, Ghosh A, Brandhorst S, et al. A diet mimicking fasting promotes regeneration and reduces autoimmunity and multiple sclerosis symptoms. Cell Rep. (2016) 15:213646. doi: 10.1016/j.celrep.2016.05.009

71. Ehrchen J, Steinmuller L, Barczyk K, Tenbrock K, Nacken W, Eisenacher $\mathrm{M}$, et al. Glucocorticoids induce differentiation of a specifically activated, anti-inflammatory subtype of human monocytes. Blood. (2006) 109:1265-74. doi: 10.1182/blood-2006-02-001115

72. Yona S, Gordon S. Inflammation: glucocorticoids turn the monocyte switch. Immunol Cell Biol. (2007) 85:81-82. doi: 10.1038/sj.icb.71 00034

73. Fingerle-Rowson G, Angstwurm M, Andreesen R, Ziegler-Heitbrock HW. Selective depletion of CD14+ CD16+ monocytes by glucocorticoid therapy. Clin Exp Immunol. (1998) 112:501-6. doi: 10.1046/j.1365-2249.1998.0 0617.x

74. Meuret G, Bremer C, Bammert J, Ewen J. Oscillation of blood monocyte counts in healthy individuals. Cell Tissue Kinet. (1974) 7:223-30. doi: 10.1111/j.1365-2184.1974.tb00902.x

75. Tak T, Drylewicz J, Conemans L, de Boer RJ, Koenderman L, Borghans JAM, et al. Circulatory and maturation kinetics of human monocyte subsets in vivo. Blood. (2017) 130:1474-7. doi: 10.1182/blood-2017-03-7 71261

76. Born J, Lange T, Hansen K, Mölle M, Fehm HL. Effects of sleep and circadian rhythm on human circulating immune cells. J Immunol. (1997) 158:4454-64.

77. Cuesta M, Boudreau P, Dubeau-Laramée G, Cermakian N, Boivin DB. Simulated night shift disrupts circadian rhythms of immune functions in humans. J Immunol. (2016) 196:2466-75. doi: 10.4049/jimmunol.15 02422

78. Lancaster GI, Khan Q, Drysdale P, Wallace F, Jeukendrup AE, Drayson MT, et al. The physiological regulation of toll-like receptor expression and function in humans. J Physiol. (2005) 563:945-55. doi: 10.1113/jphysiol.2004.081224

79. Lange T, Dimitrov S, Fehm H-L, Westermann J, Born J. Shift of monocyte function toward cellular immunity during sleep. Arch Intern Med. (2006) 16 6:1695. doi: 10.1001/archinte.166.16.1695

80. Shantsila E, Tapp LD, Wrigley BJ, Montoro-Garcia S, Ghattas A, Jaipersad A, et al. The effects of exercise and diurnal variation on monocyte subsets and monocyte-platelet aggregates. Eur
J Clin Invest. (2012) 42:832-9. doi: 10.1111/j.1365-2362.2012. 02656.x

81. McAlpine CS, Kiss MG, Rattik S, He S, Vassalli A, Valet C, et al. Sleep modulates haematopoiesis and protects against atherosclerosis. Nature. (2019) 566:383-7. doi: 10.1038/s41586-019 -0948-2

82. Lasselin J, Rehman J, Åkerstedt T, Lekander M, Axelsson J. Effect of long-term sleep restriction and subsequent recovery sleep on the diurnal rhythms of white blood cell subpopulations. Brain Behav Immun. (2015) 47:93-9. doi: 10.1016/j.bbi.2014. 10.004

83. Linden M, Larson M, Prellner T, Brattsand R, Laitinen LA. Seasonal variation in the function of blood monocytes obtained from healthy non-smokers, asymptomatic smokers, and smokers with chronic bronchitis. Chronobiol Int. (1994) 11:266-72. doi: 10.3109/07420529409067794

84. Myrianthefs P, Karatzas S, Venetsanou K, Grouzi E, Evagelopoulou P, Boutzouka E, et al. Seasonal variation in whole blood cytokine production after LPS stimulation in normal individuals. Cytokine. (2003) 24:286-92. doi: 10.1016/j.cyto.2003. 08.005

85. Shephard RJ. Development of the discipline of exercise immunology. Exerc Immunol Rev. (2010). 16:194-222.

86. Steppich B, Dayyani F, Gruber R, Lorenz R, Mack M, Ziegler-Heitbrock HWL. Selective mobilization of CD14 + CD16 + monocytes by exercise. Am J Physiol Physiol. (2000) 279:C578-86. doi: 10.1152/ajpcell.2000.279. 3.C578

87. Graff RM, Kunz HE, Agha NH, Baker FL, Laughlin M, Bigley AB, et al. $\beta 2$-Adrenergic receptor signaling mediates the preferential mobilization of differentiated subsets of CD8+ T-cells, NK-cells and non-classical monocytes in response to acute exercise in humans. Brain Behav Immun. (2018) 74:143-53. doi: 10.1016/j.bbi.2018.08.017

88. Durrer C, Francois M, Neudorf H, Little JP. Acute highintensity interval exercise reduces human monocyte Toll-like receptor 2 expression in type 2 diabetes. Am J Physiol Integr Comp Physiol. (2017) 312:R529-38. doi: 10.1152/ajpregu.0034 8.2016

89. Slusher AL, Zúñiga TM, Acevedo EO. Maximal exercise alters the inflammatory phenotype and response of mononuclear cells. Med Sci Sports Exerc. (2018) 50:675-83. doi: 10.1249/MSS.0000000000001480

90. Gabriel H, Urhausen A, Brechtel L, Müller H-J, Kindermann W. Alterations of regular and mature monocytes are distinct, and dependent of intensity and duration of exercise. Eur J Appl Physiol Occup Physiol. (1994) 69:179-81. doi: 10.1007/BF00 609414

91. Timmerman KL, Flynn MG, Coen PM, Markofski MM, Pence BD. Exercise training-induced lowering of inflammatory (CD14+CD16+) monocytes: a role in the anti-inflammatory influence of exercise? J Leukoc Biol. (2008) 84:1271-8. doi: 10.1189/jlb.0408244

92. Schefold JC, Porz L, Uebe B, Poehlmann H, Von Haehling S, Jung A, et al. Diminished HLA-DR expression on monocyte and dendritic cell subsets indicating impairment of cellular immunity in pre-term neonates: a prospective observational analysis. J Perinat Med. (2015) 43:609-18. doi: 10.1515/jpm-20 14-0226

93. Christensen RD, Jensen J, Maheshwari A, Henry E, Gastroenterology P. Reference ranges for blood concentrations of eosinophils and monocytes during the neonatal period defined from over 63000 records in a multihospital health-care system and 4 Divisions of Neonatology and. J Perinatol. (2010) 30:540-5. doi: 10.1038/jp.20 09.196

94. Franceschi C, Bonafè M, Valensin S, Olivieri F, De Luca M, Ottaviani E, De Benedicits G. Inflamm-aging. An evolutionary perspective on immunosenescence. Ann NY Acad Sci. (2000) 908:244-54. doi: 10.1111/j.1749-6632.2000.tb06651.x

95. Metcalf TU, Cubas RA, Ghneim K, Cartwright MJ, Grevenynghe J, Van Richner JM, et al. Global analyses revealed age-related alterations in innate immune responses after stimulation of pathogen 
recognition receptors. Aging Cell. (2015) 14:421-32. doi: 10.1111/acel. 12320

96. Nyugen J, Agrawal S, Gollapudi S, Gupta S. Impaired functions of peripheral blood monocyte subpopulations in aged humans. J Clin Immunol. (2010) 30:806-13. doi: 10.1007/s10875-010-9448-8

97. Ong S-M, Hadadi E, Dang T-M, Yeap W-H, Tan CT-Y, Ng T-P, et al. The pro-inflammatory phenotype of the human non-classical monocyte subset is attributed to senescence. Cell Death Dis. (2018) 9:266. doi: 10.1038/s41419-018-0327-1

98. Sadeghi HM, Schnelle JF, Thomas JK, Nishanian P, Fahey JL. Phenotypic and functional characteristics of circulating monocytes of elderly persons? Exp Gerontol. (1999) 34:959-70. doi: 10.1016/S0531-5565(99)00065-0

99. Seidler S, Zimmermann HW, Bartneck M, Trautwein C, Tacke F. Age-dependent alterations of monocyte subsets and monocyte-related chemokine pathways in healthy adults. BMC Immunol. (2010) 11:30. doi: 10.1186/1471-2172-11-30

100. Verschoor CP, Johnstone J, Millar J, Parsons R, Lelic A, Loeb M, et al. Alterations to the frequency and function of peripheral blood monocytes and associations with chronic disease in the advanced-age, frail elderly. PLoS ONE. (2014) 9:e104522. doi: 10.1371/journal.pone.0104522

101. de Pablo-Bernal RS, Cañizares J, Rosado I, Galvá MI, Alvarez-Ríos AI, Carrillo-Vico A, et al. Monocyte phenotype and polyfunctionality are associated with elevated soluble inflammatory markers, cytomegalovirus infection, and functional and cognitive decline in elderly adults. $J$ Gerontol. Ser. A Biol. Sci. Med. Sci. (2016) 71:610-8. doi: 10.1093/gerona/ glv121
102. Puchta A, Naidoo A, Verschoor CP, Loukov D, Thevaranjan N, Mandur TS, et al. TNF drives monocyte dysfunction with age and results in impaired anti-pneumococcal Immunity. PLoS Pathog. (2016) 12:e1005368. doi: 10.1371/journal.ppat.1005368

103. Menezes S, Melandri D, Anselmi G, Perchet T, Loschko J, Dubrot J, et al. The heterogeneity of Ly6Chi monocytes controls their differentiation into iNOS+ macrophages or monocyte-derived dendritic cells. Immunity. (2016) 45:1205-18. doi: 10.1016/j.immuni.2016.12.001

104. Satoh T, Nakagawa K, Sugihara F, Kuwahara R, Ashihara M, Yamane F, et al. Identification of an atypical monocyte and committed progenitor involved in fibrosis. Nature. (2017) 541:96-101. doi: 10.1038/nature20611

105. Yáñez A, Coetzee SG, Olsson A, Muench DE, Berman BP, Hazelett DJ, et al. Granulocyte-monocyte progenitors and monocyte-dendritic cell progenitors independently produce functionally distinct monocytes. Immunity. (2017) 47:890-902.e4. doi: 10.1016/j.immuni.2017.10.021

Conflict of Interest: The authors declare that the research was conducted in the absence of any commercial or financial relationships that could be construed as a potential conflict of interest.

Copyright $@ 2019$ Patel and Yona. This is an open-access article distributed under the terms of the Creative Commons Attribution License (CC BY). The use, distribution or reproduction in other forums is permitted, provided the original author(s) and the copyright owner(s) are credited and that the original publication in this journal is cited, in accordance with accepted academic practice. No use, distribution or reproduction is permitted which does not comply with these terms. 